\title{
Takayasu's disease presenting as a nephrotic syndrome due to amyloidosis
}

\author{
Ana E. Sousa, Margarida Lucas, Isabel Tavora' ${ }^{1}$ and Rui M.M. Victorino \\ University Hospital of Santa Maria, Faculty of Medicine of Lisbon, Departments of Medicine 2 and \\ ${ }^{1}$ Radiology, Lisbon, Portugal
}

\begin{abstract}
Summary: We report an 18 year old black woman who presented with nephrotic syndrome in whom the investigations led to the diagnosis of diffuse Takayasu's disease, renal amyloidosis of AA type and interstitial lung disease. Proteinuria in Takayasu's disease is usually ascribed to hypertension or more rarely to glomerulonephritis. This case suggests that amyloidosis should be considered also in the investigation of proteinuria in these patients in view of the serious prognostic implications. This case represents further evidence that Takayasu's disease can be the cause of systemic reactive amyloidosis which may also be the presenting feature.
\end{abstract}

\section{Introduction}

Takayasu's disease is a large vessel vasculitis of possible immunological aetiology and recent evidence suggests that its incidence is much higher (2.6 per million year) than previously reported. ${ }^{1}$

Amyloidosis is a well-recognized complication of idiopathic inflammatory disorders such as rheumatoid arthritis ${ }^{2}$ or Behcet's disease ${ }^{3}$ and has been described previously in Takayasu's disease. ${ }^{4-7}$

We report a case of asymptomatic Takayasu's disease where the clinical presentation was a nephrotic syndrome due to AA type amyloidosis.

\section{Case report}

An 18 year old black woman, living in Angola, was admitted to Santa Maria Hospital, Lisbon, with a nephrotic syndrome of unknown cause. Four years previously there was an episode of fever, fatigue and generalized oedema. Since then she had had several episodes of generalized oedema without fever and, one year before admission, was found to have (nephrotic range) proteinuria, weak peripheral pulses, bilateral carotid murmurs, a systolic abdominal bruit and normal blood pressure.

Physical examination on admission, however, showed no fever or peripheral oedema. The blood

Correspondence: Professor Rui M.M. Victorino, M.D. (Lisbon), Ph.D. (Lond.), Faculty of Medicine of LisbonMedicine 2, Av. Prof. Egas Moniz, 1600 Lisbon, Portugal.

Accepted: 30 November 1992 pressure was $150 / 100 \mathrm{mmHg}$ in the left arm and was unmeasurable in the right arm. All peripheral pulses were feeble and the left carotid was absent. A systolic murmur was heard in the abdomen above the umbilicus. Laboratory investigations showed haemoglobin $10.2 \mathrm{~g} / \mathrm{dl}$, white cell count $9.3 \times 10^{9} \mathrm{~g} / \mathrm{dl}$, ESR $135 \mathrm{~mm}, \mathrm{C}$-reactive proteinnegative, creatinine $100 \mu \mathrm{mol} / \mathrm{l}$ and serum albumin $13 \mathrm{~g} / \mathrm{l}$. No monoclonal proteins or free light chains were found on serum and urinary immunoelectrophoresis. Daily urinary protein varied between 3.3 and $10.5 \mathrm{~g}$. Cholesterol was $18 \mathrm{mmol} / \mathrm{l}$, and C3, C4 and $\mathrm{CH} 50$ were normal. Antinuclear antibodies were absent and serological tests for syphilis were non-reactive.

Chest X-ray showed a diffuse interstitial infiltrate and areas of hypoperfusion with nonsegmental distribution were seen in a Tc-99 lung scan. Pulmonary function tests revealed a restrictive pattern with a reduced diffusing capacity $(56 \%$ of predicted).

These findings led to the diagnosis of interstitial pulmonary disease. Percutaneous renal biopsy showed extensive amyloid deposits which stained with Congo red dye but lost the red staining characteristics and the green birefringence under polarized light when treated with potassium permanganate. Digital subtraction angiography showed irregularities of the aortic arch and multiple areas of ectasia and stenosis in the innominate artery (Figure 1). A major stenosis was seen at the origin of the left carotid artery and there were multiple irregularities of the lumen of the left 


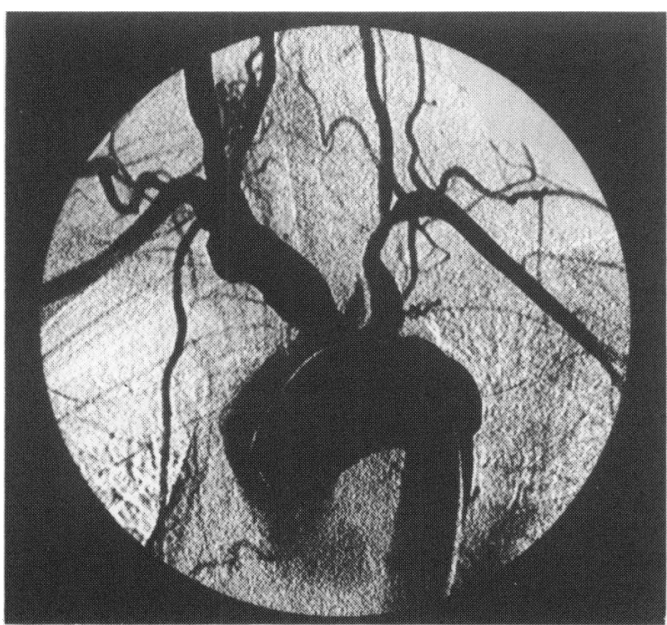

Figure 1 Digital subtraction angiography of the aortic arch.

subclavian artery. A diffuse narrowing of the abdominal aorta was also seen.

A diagnosis of Takayasu's disease associated with renal amyloidosis and interstitial pulmonary disease was made and prednisone $60 \mathrm{mg}$ daily was started. One year later her renal function began to deteriorate rapidly requiring haemodialysis and the patient died 3 months later due to sepsis.

\section{Discussion}

The patient reported here fulfills the usual clinical and angiographic criteria for Takayasu's disease, but is remarkable because the first clinical manifestation was a nephrotic syndrome due to renal amyloidosis. The fact that the renal amyloid was sensitive to potassium permanganate suggests that it is of AA type as expected in reactive amyloidosis and, in this context, it is important to stress that the

\section{References}

1. Hall, S., Barr, W., Lie, J.T., Stanson, A.W., Kazmier, F.J. \& Hunder, G.G. Takayasu arteritis: a study of 32 North American patients. Medicine 1985, 64: 89-99.

2. Husby, G. Amyloidosis in rheumatoid arthritis. Ann Clin Res 1975, 7: 154-167.

3. Beroniade, V. Amyloidosis and Behcet's disease. Ann Intern Med 1975, 83: 904-905.

4. Dash, S.C., Sharma, R.K., Malhotra, K.K. \& Bhuyan, U.N. Renal amyloidosis and non-specific aorto-arteritis - a hitherto undescribed association. Postgrad Med J 1984, 60: 626-628.

5. Graham, A.N., Delahunt, B., Renouf, J.J. \& Austad, W.I. Takayasu's disease associated with generalised amyloiditis. Aust NZ J Med 1985, 15: 343-345.

6. Matsuda, M., Makayama, J., Haruta, S., Tsukada, N., Yanagisawa, N. \& Furukawa, T. A case of Takayasu's arteritis associated with nephrotic syndrome, aortic regurgitation and amyloidosis. Nippon Naika Gakkai Zasshi 1987 , 76: $832-836$. usual causes of reactive (secondary) amyloidosis were excluded. It is possible to postulate that mechanisms similar to those involved in the production of amyloid in idiopathic inflammatory disorders such as rheumatoid arthritis may also occur in Takayasu's disease. This case represents further evidence in favour of this association recently described ${ }^{4-7}$ and illustrates the possibility of a nephrotic syndrome due to renal amyloidosis as a form of clinical presentation of Takayasu's disease.

Proteinuria in Takayasu's disease is a frequent occurrence and it is usually attributed to hypertensive nephropathy and more rarely to glomerulonephritis. ${ }^{8,9}$ In view of the prognostic implications of renal amyloidosis in the context of a systemic vasculitis, it is important to consider this possibility in the investigation of proteinuria in patients with Takayasu's disease. As this vasculitis may evolve asymptomatically for long periods, it is also important to consider the diagnosis of Takayasu's disease in the investigation of secondary amyloidosis.

The pulmonary manifestations of Takayasu's disease are usually due to pulmonary artery involvement and are thought to occur in as many as $50 \%$ of the cases. ${ }^{10}$ Although interstitial lung disease occurs in several systemic vasculitis, its recognition in Takayasu's disease is limited to a single case report. ${ }^{9}$ The investigations in our patient are consistent with interstitial lung disease similar to the case report by Greene et al. ${ }^{9}$

In conclusion, this case favours the view that systemic reactive amyloidosis can be caused by Takayasu's disease and may be the presenting feature.

\section{Acknowledgements}

We are grateful to Professor Carneiro de Moura, Director of the Department of Medicine 2; to Dr A. Gomes da Costa and Professor Diniz da Gama for clinical discussion; and to Professor A. Duarte and Dr Peixoto Menezes for the pathological study.

7. Van der Meulan, J., Gupta, R.K., Peregrin, J.H., Al Adnani, M.S. \& Johny, K.V. Takayasu's arteritis and nephrotic syndrome in a patient with crossed renal ectopia. Neth J Med 1989, 34: 142-147.

8. Shelhamer, J.H., Volkman, D.J., Parrillo, J.E., Lawley, T.J., Johnston, M.R. \& Fauci, A.S. Takayasu's arteritis and its therapy. Ann Intern Med 1985, 103: 121-126.

9. Greene, N.B., Baughman, R.P. \& Kim, C.K. Takayasu's arteritis associated with interstitial lung disease and glomerulonephritis. Chest 1986, 89: 605-606.

10. Leavitt, R.Y. \& Faucy, A.S. Pulmonary vasculitis. Am Rev Respir Dis 1986, 134: 149-166. 